\title{
Sparse Tucker Tensor Decomposition on a Hybrid FPGA-CPU Platform
}

\author{
Weiyun Jiang, Kaiqi Zhang, Colin Yu Lin, Feng Xing, Zheng Zhang, Member, IEEE
}

\begin{abstract}
Recommendation systems, social network analysis, medical imaging, and data mining often involve processing sparse high-dimensional data. Such high-dimensional data are naturally represented as tensors, and they cannot be efficiently processed by conventional matrix or vector computations. Sparse Tucker decomposition is an important algorithm for compressing and analyzing these sparse high-dimensional data sets. When energy efficiency and data privacy are major concerns, hardware accelerators on resource-constraint platforms become crucial for the deployment of tensor algorithms. In this work, we propose a hybrid computing framework containing CPU and FPGA to accelerate sparse Tucker factorization. This algorithm has three main modules: tensor-times-matrix (TTM), Kronecker products, and $Q R$ decomposition with column pivoting (QRP). In addition, we accelerate the former two modules on a Xilinx FPGA and the latter one on a CPU. Our hybrid platform achieves $23.6 \times \sim 1091 \times$ speedup and over $93.519 \% \sim 99.514 \%$ energy savings compared with CPU on the synthetic and real-world datasets.
\end{abstract}

\section{INTRODUCTION}

As massive data is collected from social media, wearable devices and internet of things, novel algorithms and platforms are highly desired to handle data-intensive computing tasks. Vector- and matrix-based methods can efficiently process 1way data (e.g., a sequence of voice data) or 2-way data (e.g., a gray-scale image), but they are often inefficient to handle multi-way data. Representative examples includes 3way (or order-3) E-commerce data (which records customers' preference on massive products over a few months), 4-way (or order-4) cardiac image data (which records the spatial data of $3 \mathrm{D}$ at multiple time points). Processing such multi-way data often suffers from the curse of dimensionality.

Tensors are a high-order generalization of matrices and vectors, and they are a natural tool to represent and process multi-way data [1]. Leveraging various tensor decomposition or factorization methods [1]-[4], the curse of dimensionality of storing and computing multi-way data can be avoided or significantly mitigated in many applications. For instance, the canonical polyadic (CP) [5], [6] and tensor-train [2] factorizations can reduce the storage cost and unknown variables from an exponential function to a linear one. Tucker factorization [3] can be used for high-order principle component analysis or facial recognition [7]-[9]. Tensor computation has achieved tremeonduous success in data mining [10], computer vision [7]-[9], medical imaging [11], electronic design automation [12]-[15] and deep learning [16]-[18].

The emerging tensor computation concept brings in massive research opportunities and challenges on the hardware level. Due to the fundamental difference between tensor and matrix computations, we may need to re-think many

This work was supported by NSF Award 1817037.

W. Jiang, K. Zhang and Z. Zhang are with the the Department of Electrical and Computer Engineering, University of California, Santa Barbara CA 93106, USA (e-mail: weiyunjiang@ucsb.edu, kzhang70@ucsb.edu, and zhengzhang@ece.ucsb.edu).

C. Y. Lin and F. Xing are with Xilinx Inc., Beijing, China (e-mail: YULIN1@xilinx.com and FENGX@xilinx.com). aspects of tensor computation (e.g., storage, computing and data movement) on specific platforms. Increasing research results have been reported to improve the tensor data storage and computing on the cloud and high-performance clusters [19]-[21]. However, little work has been done on resourceconstrained platforms. This becomes increasingly important as the need of energy-efficient machine learning and data privacy surges. In order to address this issues, some efforts have been made towards tensor-compressed neural networks on mobile devices [22] and dense tensor operations on FPGA. For instance, some dense tensor operations including MTTKRP, TTM and TTMc were accelerated in [23]; a spectral analysis of Hankel tensors was reported in [24]. To perform dense Tucker decomposition on FPGA, Zhang et al. [25] divided the hardware architectures into three modules: tensor-timesmatrix, singular value decomposition via Jacobi iterations and tensor permutation/reshaping. In addition, a warm-start algorithm was used to reduce the cost of Jacobi iterations. The resulting FPGA accelerator demonstrated significant speedup compared with both CPU and GPU. However, the FPGA accelerator [25] cannot exploit data sparsity, and it becomes energy- and time-inefficient when dealing with sparse tensors. Ref. [26] reported some sparse tensor computation kernels. For instance, it demonstrated how to implement both dense and sparse tensor operations, such as sparse TTMc via sparse compute pattern $S F^{3}$. To our best knowledge, there is no FPGA accelerator available for sparse Tucker decomposition.

In this paper, we investigate the hardware acceleration of Tucker factorization for sparse tensor data. Sparse tensors widely appear in practice due to the missing information in recommendation systems, medical image or E-commerce data. For instance, in magnetic resonance imaging (MRI), one can generate a sparse tensor by partial MRI scanning, then reconstruct the whole image with a low cost [27]. In neuroscience, researchers use sparse tensors to monitor the brain variability [28]. In EDA, it is often too expensive to obtain all simulation or measurement data, thus one uses a partially sampled sparse tensor for process variation or performance uncertainty prediction [12], [14], [15]. Although extensive algorithms have been developed to process sparse tensors, their hardware/algorithm co-optimization remains a rarely explored field [25]. This task has become increasingly important as energy efficiency and privacy cause lots of concerns in the data science and machine learning community.

\section{A. Paper Contributions and Organization}

This paper proposes to design an energy- and memoryefficient hybrid FPGA-CPU accelerator for sparse Tucker decomposition [19]. This algorithm consists of three major components: tensor-times-matrix (TTM) [1], Kronecker product [29] and QR decomposition with column pivoting (QRP) [30]. Our specific contributions include:

- On the hardware side, we present a high-level synthesis (HLS) FPGA implementation for sparse Tucker decomposition. We describe the design of two modules, TTM 

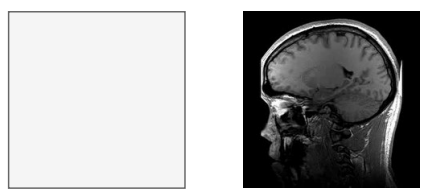

(a) Matrices

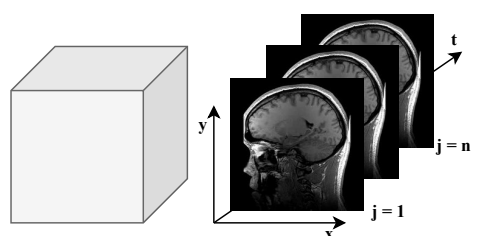

(b) 3-Way Tensors

Fig. 1. (a) A matrix is a 2-D data array (e.g., one slice of MRI data), (b) a 3-way tensor is a 3-D data array (e.g., multiple slices of images).

and Kronecker product, by exploiting the data sparsity.

- On the algorithm side, we replace the conventional singular value decomposition (SVD) [31] with the QR decomposition with column pivoting (QRP) [30] to reduce the data storage cost and to speed up the computation.

- We implement our FPGA accelerator in a Xilinx FPGA on Amazon web service (AWS). Then we compare our hybrid FPGA-CPU accelerator with CPU and with the recently developed dense FPGA accelerator [25] on synthetic and real-world sparse tensor benchmarks. Our hybrid FPGA-CPU accelerator achieves $1.15 \times \sim 1091 \times$ speedup and consumes $93.519 \% \sim 99.514 \%$ less energy. In addition, our proposed accelerator achieves significant speedup $(23.6 \times \sim 1091 \times)$ when the tensor is very large and sparse

This paper is organized as follows. Section II introduces some background information about tensor operations. Section III presents the algorithm and our Vivado HLS FPGA design of a sparse Tucker decomposition. We compare our FPGA/CPU hybrid platform with CPU and the dense Tucker FPGA accelerator [25] in terms of run-time and energy efficiency in Section IV Finally, Section V concludes this paper.

\section{PReliminaries of TENSORS}

This section presents some background about tensors, which is necessary for understanding the ideas of this paper.

Definition 1: A tensor $\mathcal{X} \in \mathbb{R}^{I_{1} \times I_{2} \times \cdots \times I_{N}}$ is a highdimensional array of order $N$. Here the order $N$ (also known as "way") is the total number of dimensions. A matrix $\mathbf{X} \in$ $\mathbb{R}^{n_{1} \times n_{2}}$ is a 2 nd-order (or 2-D) tensor, and its element indexed by $\left(i_{1}, i_{2}\right)$ can be denoted as $x_{i_{1} i_{2}}$. For a general $N$ th-order (or $N$-way) tensor $\mathcal{X}$, its element indexed by $\left(i_{1}, i_{2} \cdots, i_{N}\right)$ is denoted as $x_{i_{1} i_{2} \cdots i_{N}}$.

Fig. 1 shows a matrix (e.g., one slice of MRI data) and a 3-way tensor, respectively. In this paper, we use boldface lower-case letters (e.g., x) to denote vectors, boldface uppercase letters (e.g., $\mathbf{X}$ ) to denote matrices, and boldface Euler script letters ( e.g., $\mathcal{X}$ ) to denote tensors. A scalar is denoted by a lower-case letter, e.g., $x$.

Definition 2: The inner product of two tensors with the same size is defined as

$$
\langle\mathcal{X}, \mathcal{Y}\rangle=\sum_{i_{1} i_{2} \cdots i_{N}} x_{i_{1} i_{2} \cdots i_{N}} y_{i_{1} i_{2} \cdots i_{N}}
$$

Furthermore, the Frobenius norm (also known as F-norm) of a tensor $\mathcal{X}$ is defined as $\|\mathcal{X}\|_{\mathrm{F}}=\sqrt{\langle\mathcal{X}, \mathcal{X}\rangle}$.

Definition 3: A matricization operation, (also known as unfolding or flattening), reshapes a tensor into a matrix. The mode- $n$ matricization of a tensor $\mathcal{X} \in \mathbb{R}^{I_{1} \times I_{2} \times \cdots \times I_{N}}$ is denoted as $\mathbf{X}_{(n)}$ which has $I_{n}$ rows and $\prod_{k \neq n} I_{k}$ columns.

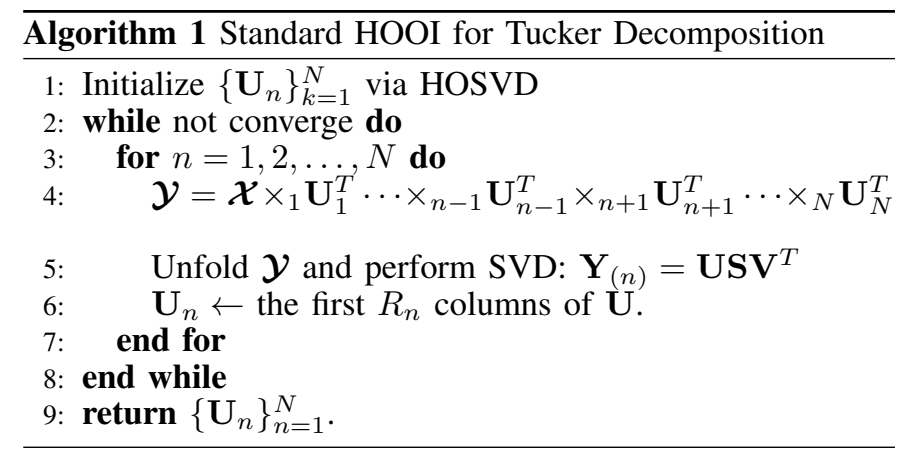

Element-wise, we have each entry of $\mathbf{X}_{(n)}$ as

$\mathbf{X}_{(n)}\left(i_{n}, j\right)=x_{i_{1} i_{2} \cdots i_{N}}$ with $j=1+\sum_{k=1, k \neq n}^{N}\left(i_{k}-1\right) \prod_{m=1, m \neq n}^{k-1} I_{m}$.

Definition 4: The mode-n tensor matrix product [or tensortimes-matrix (TTM)], between a tensor $\mathcal{X} \in \mathbb{R}^{I_{1} \times I_{2} \times \cdots \times I_{N}}$ and a matrix $\mathbf{U} \in \mathbb{R}^{J \times I_{n}}$ is denoted as

$$
\mathcal{G}=\mathcal{X} \times{ }_{n} \mathbf{U}, \text { where } \mathcal{G} \in \mathbb{R}^{I_{1} \times \cdots \times I_{n-1} \times J \times I_{n+1} \times \cdots \times I_{N}} .
$$

Element-wise, we can write this operation as

$$
g_{i_{1} \cdots n-1} j i_{n+1} \ldots i_{N}=\sum_{i_{n}=1}^{I_{n}} x_{i_{1} i_{2} \ldots i_{N}} u_{j i_{n}} .
$$

We may also obtain a TTM product by using the unfolded tensors:

$$
\mathcal{G}=\mathcal{X} \times_{n} \mathbf{U} \Leftrightarrow \mathbf{G}_{(n)}=\mathbf{U X}_{(n)} .
$$

We further introduce a matrix operation that will be used in our subsequent tensor computation.

Definition 5: Given a matrix $\mathbf{A} \in \mathbb{R}^{m \times n}$ and another matrix $\mathbf{B} \in \mathbb{R}^{p \times q}$, their Kronecker product $\mathbf{A} \otimes \mathbf{B}$ is the following matrix $\mathbf{C} \in \mathbb{R}^{m p \times n q}$

$$
\mathbf{C}=\mathbf{A} \otimes \mathbf{B}=\left[\begin{array}{ccc}
a_{11} \mathbf{B} & \cdots & a_{1 n} \mathbf{B} \\
\vdots & \ddots & \vdots \\
a_{m 1} \mathbf{B} & \cdots & a_{m n} \mathbf{B}
\end{array}\right]
$$

\section{ACCELERATOR FOR SPARSE TUCKER DECOMPOSITION}

Given a tensor $\mathcal{X} \in \mathbb{R}^{I_{1} \times I_{2} \times \cdots \times I_{N}}$, the Tucker decomposition [4] approximates it with a small low-rank core tensor $\mathcal{G} \in$ $\mathbb{R}^{R_{1} \times R_{2} \times \cdots \times R_{N}}$ and $N$ factor matrices $\left\{\mathbf{U}_{n} \in \mathbb{R}^{I_{n} \times R_{n}}\right\}_{n=1}^{N}$ :

$$
\mathcal{X} \approx \mathcal{G} \times{ }_{1} \mathbf{U}_{1} \times_{2} \mathbf{U}_{2} \cdots \times_{N} \mathbf{U}_{N}
$$

Here $\left(R_{1}, R_{2}, \cdots, R_{N}\right)$ is a multilinear tensor rank. 

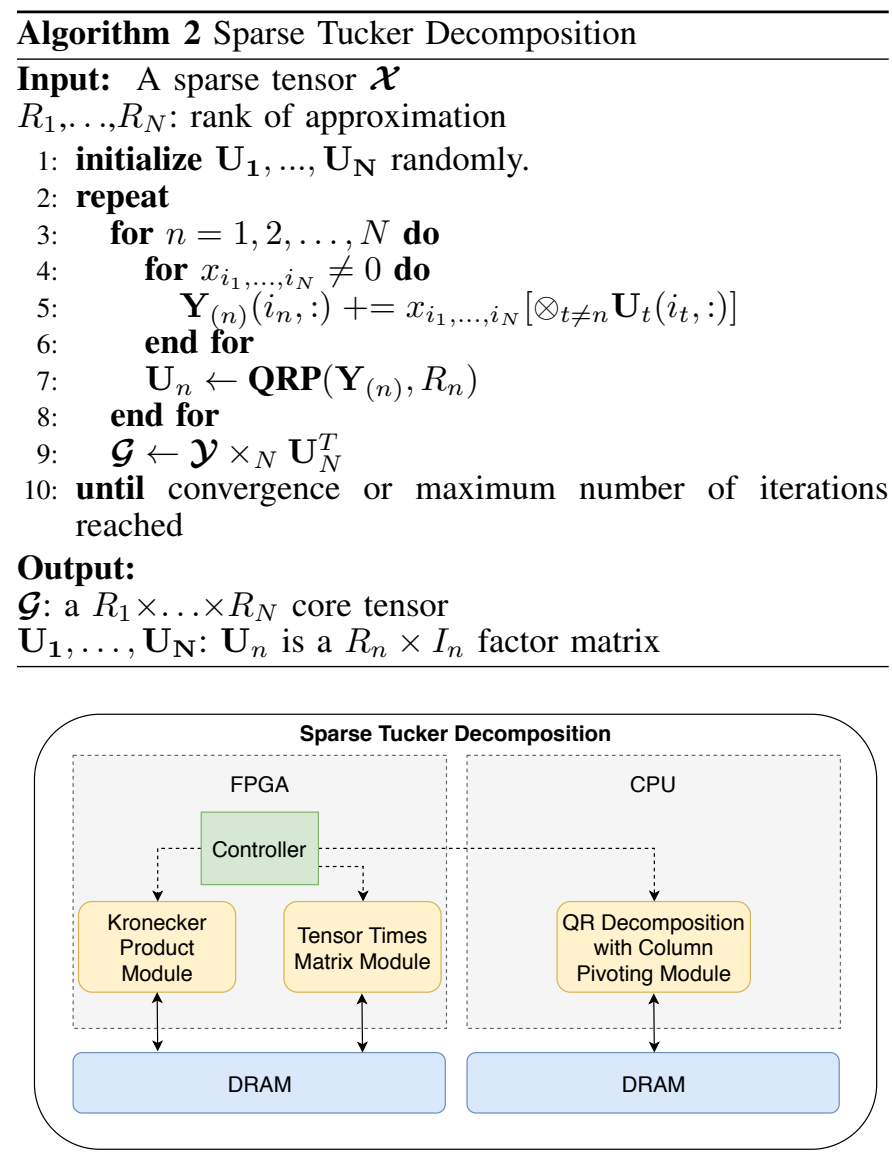

Fig. 2. A Hybrid FPGA-CPU platform for sparse Tucker factorization.

The Tucker decomposition can be regarded as a highorder generalization of singular value decomposition (SVD), and it is often implemented with the power iteration method called high-order orthogonal iteration (HOOI) in [4]. As shown in Alg. 1, it aims to find the orthogonal matrices $\left\{\mathbf{U}_{n} \in \mathbb{R}^{I_{n} \times R_{n}}\right\}_{n=1}^{N}$ to maximize the F-norm of

$$
\mathcal{G}=\mathcal{X} \times{ }_{1} \mathbf{U}_{1}^{T} \times{ }_{2} \mathbf{U}_{2}^{T} \cdots \times{ }_{N} \mathbf{U}_{N}^{T} .
$$

In every iteration, we need to compute the $R_{n}$ dominant left singular vectors of unfolded matrix $\mathbf{Y}_{(n)}$, where

$$
\mathcal{Y}=\mathcal{X} \times{ }_{1} \mathbf{U}_{1}^{T} \cdots \times{ }_{n-1} \mathbf{U}_{n-1}^{T} \times_{n+1} \mathbf{U}_{n+1}^{T} \cdots \times{ }_{N} \mathbf{U}_{N}^{T} .
$$

The orthogonal matrix is obtained by a SVD of the unfolded matrix $\mathbf{Y}_{(n)}$.

The standard HOOI becomes very inefficient for sparse tensors because Line 4 of Alg. 11 does not exploit any data sparsity and always performs $N-1$ times of TTM operations.

\section{A. Overall Algorithm Flow}

In this paper, we design an FPGA-CPU hybrid accelerator based on [19] to perform Tucker factorization for sparse tensors. Two formats can be used to represent sparse tensors:

- The coordinate format (COO) stores a sparse tensor with all nonzero elements and their associated coordinate vectors, shown in Table $\mathrm{I}$. The first four columns represent the coordinate $(i, j, k, l)$ of 4 nonzero elements, and the last column represents the corresponding value. The $\mathrm{COO}$ format usually requires storage of $O(n n z * N)$ index values and $O(n n z)$ nonzero data values, where $n n z$ is
TABLE I

COORDINATE (COO) FORMAT OF A $5 \times 5 \times 5 \times 5$ SPARSE TENSOR. HERE $(i, j, k, l)$ DENOTES AN INDEX, AND $n n z$ IS THE VALUE OF AN ASSOCIATED NON-ZERO DATA ELEMENT.

\begin{tabular}{|c|c|c|c|c|}
\hline$i$ & $j$ & $k$ & $l$ & $n n z$ \\
\hline 1 & 1 & 1 & 1 & 2 \\
\hline 1 & 1 & 1 & 5 & 7.5 \\
\hline 1 & 1 & 3 & 5 & 4 \\
\hline 2 & 2 & 2 & 4 & 5 \\
\hline
\end{tabular}

the number of nonzero elements and $N$ is the mode of the tensor.

- Compressed sparse fiber format (CSF) stores a sparse tensor by compressing the indices of nonzero elements that share the same coordinates. It is regarded as high dimensional version of the compressed sparse row (CSR) or compressed sparse column (CSC) formats used for matrices in [32]. The CSF format requires $O(2 *(n n z+$ $s+f)+2$ ) to store an order-3 tensor with $s$ slices, $f$ fibers and $n n z$ non-zero values.

In this paper, we use the COO format because of its flexibility and simplicity. Furthermore, the $\mathrm{COO}$ format provides better performance on merging-related TTM [33]. If we do not assume any special structure of the tensor and the nonzero elements are uniformly distributed, there will be rarely multiple nonzero elements in a given fiber. In such a general case, the CSF format barely has any advantages in storage compression.

The algorithm flow is summarized in Alg. 2. Compared with the standard dense Tucker factorization, the following techniques are used to exploit the data sparsity:

- Instead of storing the whole tensor, we only store the nonzero entries by specifying their values and indices.

- When performing the tensor-times-matrix (TTM) in (9), we do not perform $N-1$ levels of iterations over all modes except mode $n$. Instead, we only consider the nonzero elements of $\mathcal{X}$ and have a one-level iteration over the indices of all non-zero elements in $\mathcal{X}$.

- In order to reduce the computational and memory cost of extracting orthogonal matrix factor $\mathbf{U}_{n}$, we replace the SVD of $\mathbf{Y}_{(n)}$ with a QR decomposition with column pivoting (QRP).

The proposed accelerator architecture is shown in Fig. 2 Because it is difficult to parallelize the QRP operation, we implement it on CPU. Both (8) and (9) require TTM operations, but they are handled in different ways. For (8) we only need to compute

$$
\mathcal{G}=\mathcal{Y} \times{ }_{N} \mathbf{U}_{N}
$$

once for each iteration after obtaining $\mathcal{Y}$ (which is often dense) by (9). Therefore, we design a specialized TTM module on FPGA in Section III-B. For the power iteration in (9), we design a Kronecker product module on FPGA to accelerate the sparse operation, which is detailed in Section III-C

\section{B. Tensor-Times-Matrix (TTM) on FPGA}

The computation of $\mathcal{G}$ in $(8)$ requires $N$ tensor-matrix products on the original huge-size tensor $\mathcal{X}$. This expensive computation actually can be simplified.

Assuming that we have already done the power iteration 9 for $n=N$, and obtained a small-size tensor $\mathcal{Y} \in \mathbb{R}^{R_{1} \times R_{2} \times \cdots \times I_{N}}$ and an orthogonal factor matrix $\mathbf{U}_{N} \in$ $\mathbb{R}^{I_{N} \times R_{N}}$. We only need to compute the mode- $N$ tensor-matrix 

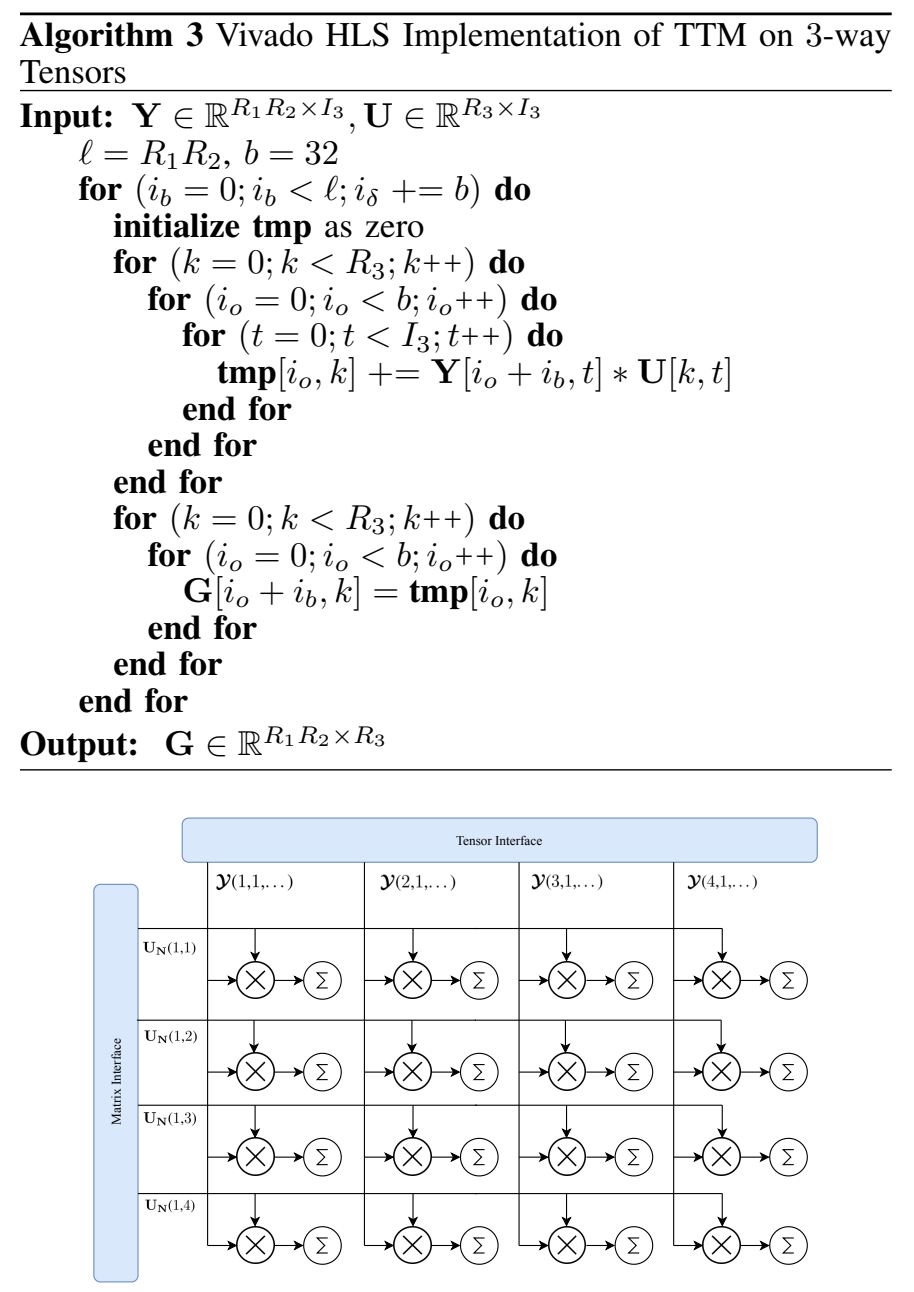

Fig. 3. Tensor-times-matrix (TTM) data flow.

product $(10)$ to obtain the core tensor $\mathcal{G}$ (line 9 , Alg. 2). This TTM can be written in an element-wise manner:

$$
\left(\mathcal{Y} \times_{N} \mathbf{U}_{N}^{T}\right)_{r_{1} r_{2} \ldots r_{N}}=\sum_{i_{N}=1}^{I_{N}} y_{r_{1} r_{2} \ldots i_{N}} \mathbf{U}_{N}\left(i_{N}, r_{N}\right)
$$

Equivalently, we can express this particular TTM with unfolded tensors as follows:

$$
\mathcal{G}=\mathcal{Y} \times_{N} \mathbf{U}_{N}^{T} \Leftrightarrow \mathbf{G}_{(N)}=\mathbf{U}_{N}^{T} \mathbf{Y}_{(N)}
$$

Here $\mathcal{G}_{(N)}$ and $\mathbf{Y}_{(N)}$ are the mode- $N$ unfolding of the tensors $\mathcal{G}$ and $\mathcal{Y}$, respectively.

In FPGA design, the 3 -D sparse tensor $\mathcal{X} \in \mathbb{R}^{I_{1} \times I_{2} \times I_{3}}$ is stored with a cost $O(n n z)$, where $n n z$ denotes the number of nonzero elements. However, the tensor $\mathcal{Y} \in \mathbb{R}^{R_{1} \times R_{2} \times I_{3}}$ in 10 is dense, and we need to store all of its elements. Although $\mathcal{Y}$ is multi-dimensional, it is unnecessary to create a new copy of this tensor. We can just reshape it into a 2-D matrix of size $R_{1} R_{2} \times I_{3}$. Meanwhile, it is critical to process the entries of $\mathcal{Y}$ in several batches. The batch size, $b$, controls the number of entries in $\mathcal{Y}$, being processed in each iteration. If we set the batch size as $b=R_{1} R_{2}$, we will end up with 3 nested for-loops because the outermost for-loop is redundant. As a result, all the entries of $\mathcal{Y}$ have to be processed at the same time, resulting in an extremely large amount of loop unrolling, which is not practical when $R_{1} R_{2}$ is larger. To overcome this issue, we decrease our batch size to 32 , and

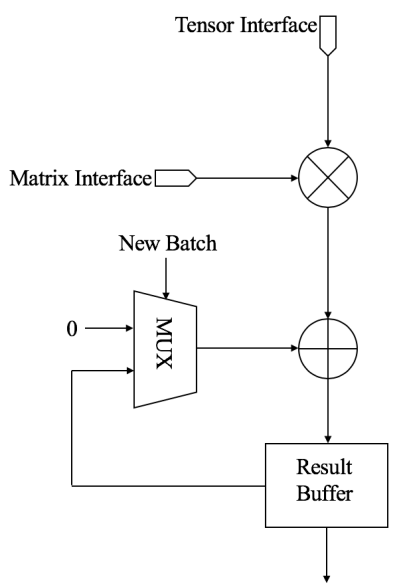

Fig. 4. Tensor-times-matrix (TTM) Processing Element (PE) [25].

separate this loop into two parts, resulting in 4 nested for-loops to compute the resultant tensor of the TTM. In this way, we could achieve optimal loop unrolling on memory-constrained FPGAs.

We provide the Vivado HLS implementation pseudo code of the TTM for a 3-way tensor $\mathcal{X}$ in Alg. 3 Given a 3way tensor, $\mathcal{X} \in \mathbb{R}^{I_{1} \times I_{2} \times I_{3}},(10)$ is a mode- 3 TTM between $\mathcal{Y} \in \mathbb{R}^{R_{1} \times R_{2} \times I_{3}}$ and $\mathbf{U} \in \mathbb{R}^{I_{3} \times R_{3}}$, where $\mathcal{G} \in \mathbb{R}^{R_{1} \times R_{2} \times R_{3}}$ is the result. In the pseudo code, we reshape our tensors $\mathcal{Y} \in \mathbb{R}^{R_{1} \times R_{2} \times I_{3}}$ and $\mathcal{G} \in \mathbb{R}^{R_{1} \times R_{2} \times R_{3}}$ into matrices $\mathbf{Y} \in$ $\mathbb{R}^{R_{1} R_{2} \times I_{3}}$ and $\mathbf{G} \in \mathbb{R}^{R_{1} R_{2} \times R_{3}}$. Basically, we divide our result, $\mathbf{G}$, into several portions such that we can update one portion of $\mathbf{G}$ in each batch:

- First, we initialize the temporary matrix, tmp as zero matrix of size $b \times R_{3}$, where $b$ is the batch size. This temporary matrix stores one portion of our result $\mathbf{G}$.

- Then, we compute TTM by multiplying unfolded tensor $\mathbf{Y}$ and $\mathbf{U}$ based on (12) and store the results in tmp.

- Finally, we just update one portion of $\mathbf{G}$ with tmp.

In order to optimize the Vivado HLS implementation, we reshape $\mathbf{U}$ in cyclic forms by a factor of 8 , and we reshape $\mathbf{Y}$ and tmp in cyclic forms by a factor of 16 . Furthermore, in order to save RAM usage, we assign only one port of RAM to the variables, $\mathbf{Y}, \mathbf{U}$, and tmp. We also assign the intermediate variable tmp to registers instead of memory to minimize memory usage.

Fig. 3 shows the data flow in the TTM computation module on FPGA. According to the element-wise formula (11), each entry of the resultant tensor can be recognized as the sum of product between the entries from the original tensor $\mathcal{Y}$ and the entries from the matrix $\mathbf{U}_{\mathbf{N}}$. In Fig. 3. it shows that data from the tensor interface, $y_{r_{1} r_{2} \ldots i_{N}}$ multiplies with the data from the matrix interface, $\mathbf{U}_{\mathbf{N}}\left(i_{N}, r_{N}\right)$. After the multiplication, the results are summed up to obtain the entries in the resultant tensor, $\left(\mathcal{Y} \times_{N} \mathbf{U}_{N}^{T}\right)_{r_{1} r_{2} \ldots r_{N}}$.

A detailed data flow of the PE for TTM is shown in Fig. 4] which was proposed in [25]. A buffer temporarily stores the intermediate result after multiplying the tensor and the matrix. For each batch, the multiplexer selects and adds the intermediate result to the new product. Once all batches are processed, the final result is stored the DRAM.

\section{Kronecker Products on FPGA}

The power iteration (9) requires $O\left(R^{d} \times n\right)$ operations, and it consumes most of the computational power and run-time in 


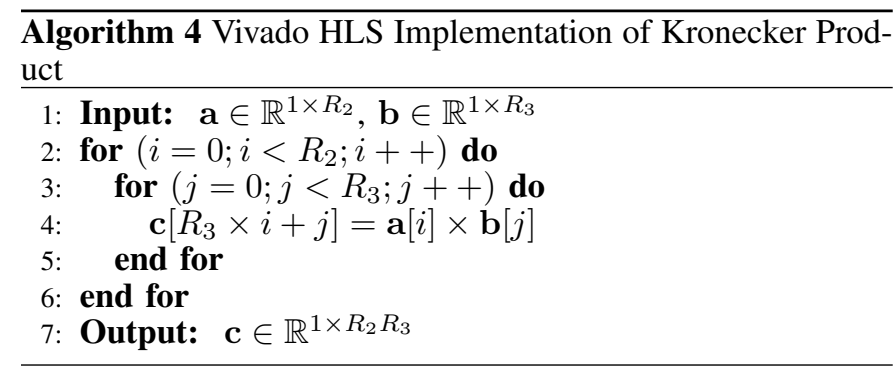

the sparse Tucker decomposition. Although an FPGA design was presented in [25] to accelerate power iterations, existing design cannot handle sparse tensor data efficiently. Therefore, leveraging [19], [29], we design an FPGA module to compute the power iteration via Kronecker products.

We consider a sparse 3 -way tensor $\mathcal{X}$ as an example. We investigate the power iteration of mode 1 , which is written as $\mathcal{Y}=\mathcal{X} \times{ }_{2} \quad \mathbf{U}_{2}{ }^{T} \times{ }_{3} \mathbf{U}_{\mathbf{3}}{ }^{T}$. To exploit the sparsity, we may choose to compute the Kronecker products and consider only nonzero elements $x_{i j k} \neq 0$ [19]:

$$
\mathbf{Y}_{(\mathbf{1})}(i,:)=\mathbf{Y}_{(\mathbf{1})}(i,:)+x_{i j k}\left[\mathbf{U}_{\mathbf{2}}(j,:) \otimes \mathbf{U}_{\mathbf{3}}(k,:)\right] \text {. }
$$

The number of Kronecker products depends on the number of nonzero elements in $\mathcal{X}$, which is often very small for sparse tensors. Furthermore, a Kronecker product can be re-used for all non-zero elements that share the same indices $(j, k)$ for the 2nd and 3rd modes. Therefore, replacing TTM of (9) with some Kronecker products can largely reduce the computational complexity. Additionally, directly computing TTM is memoryinefficient when the size and order of $\mathcal{X}$ are large, causing a high cost of RAM and registers on FPGA.

In the Vivado HLS implementation, we utilize nested forloops to implement the Kronecker product (Alg. 4):

- In order to parallelize the Kronecker product on FPGA, we pipeline the first for-loop and unroll the second for-loop. The rank of approximation, $R_{1}, R_{2}$, and $R_{3}$, are usually very small compared with the mode sizes. Therefore, the available memory, lookup tables (LUTs) and registers are often sufficient for parallelization.

- To update the corresponding rows of unfolded data $\mathbf{Y}_{(n)}$ in the the power iteration, we simply multiply the Kronecker product result in the LUTs with the corresponding nonzero element $y_{r_{1} r_{2} \ldots i_{N}}$.

- In addition, different nonzero elements may share the same index of some modes. In this case, we accumulate the multiplications between these nonzero elements and their corresponding Kronecker product results.

Fig. 5 shows the data flow inside our Kronecker product module on FPGA. To begin with, the indices of the non-zero elements in the original tensor are extracted. Then, based on the indices of the nonzero entries, the corresponding rows of the orthogonal matrix factor, $\mathbf{U}_{t}\left(i_{t},:\right)$ are selected. Assuming there are two row vectors, every entry in one row vector multiply with every entry in the other row vector to generate the Kronecker product. Since we only compute the Kronecker product between two row vectors (not two matrices), the module only requires multiplication units (no addition units).

\section{QR Decomposition with Column Pivoting}

In existing dense and sparse Tucker factorization [4], [19], the orthogonal matrix $\mathbf{U}_{n}$ is obtained with a singular value decomposition (SVD) [31] of $\mathbf{Y}_{(n)}$. The SVD is accurate but

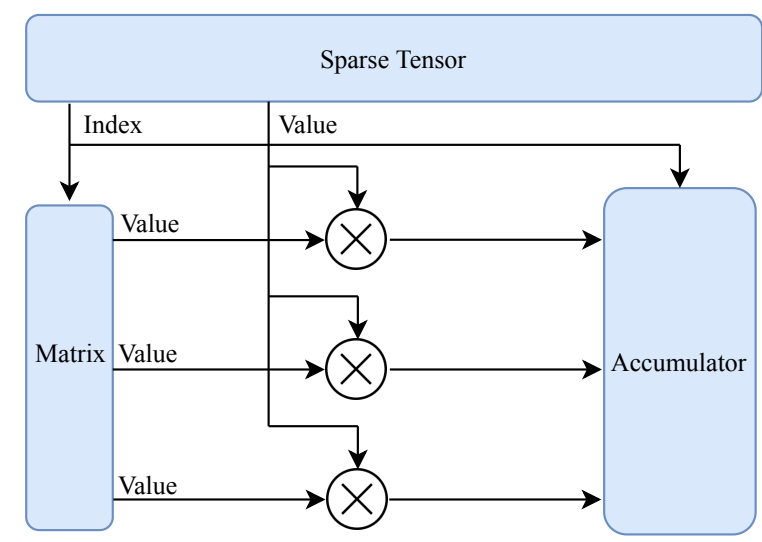

Fig. 5. The data flow of a Kronecker product.

TABLE II

ACCURACY COMPARISON OF TUCKER DECOMPOSITION WITH SVD AND WITH QRP.

\begin{tabular}{|c|c|c|}
\hline Tensor Size & $\begin{array}{c}\text { Tucker Decomposition } \\
\text { with SVD }\end{array}$ & $\begin{array}{c}\text { Tucker Decomposition } \\
\text { with QRP }\end{array}$ \\
\hline $50 \times 50 \times 50$ & $1.9222 \times 10^{-09}$ & $1.9228 \times 10^{-09}$ \\
\hline $100 \times 100 \times 100$ & $1.3846 \times 10^{-09}$ & $1.3820 \times 10^{-09}$ \\
\hline $200 \times 200 \times 200$ & $1.1588 \times 10^{-09}$ & $1.1786 \times 10^{-09}$ \\
\hline $400 \times 400 \times 400$ & $1.2114 \times 10^{-09}$ & $1.2115 \times 10^{-09}$ \\
\hline $800 \times 800 \times 800$ & $3.8450 \times 10^{-10}$ & $3.8531 \times 10^{-10}$ \\
\hline
\end{tabular}

extremely slow at computing the orthogonal matrices. In order to speed up the computation and minimize the memory usage, we propose to use $\mathrm{QR}$ decomposition with column pivoting (QRP) [30] to obtain $\mathbf{U}_{n}$. The QRP implementation does not lose any accuracy compared with the SVD implementation. This is clearly shown in Table II], which reports the errors of several low-rank Tucker decomposition with both SVD and QRP implementations, respectively.

Given a matrix $\mathbf{A} \in \mathbb{R}^{m \times n}$, the $\mathrm{QRP}$ get an orthogonal matrix $\mathbf{Q} \in \mathbb{R}^{m \times n}$ and an upper-triangular matrix $\mathbf{R} \in \mathbb{R}^{n \times n}$ :

$$
\mathbf{A P}=\mathbf{Q R},
$$

with $\mathbf{P}$ being a permutation matrix. The $\mathbf{P}$ is chosen so that the diagonal elements of $\mathbf{R}$ is non-increasing:

$$
\left|r_{11}\right| \geq\left|r_{22}\right| \geq \cdots \geq\left|r_{n n}\right| \text {. }
$$

A QRP costs about $2 m n^{2}-2 n^{3} / 3$ flops, and an SVD costs about $2 m n^{2}+11 n^{3}$ flops, where $m \geq n$. In the sparse Tucker factorization of a tensor $\mathcal{X} \in \mathbb{R}^{I_{1} \times I_{2} \times \cdots \times I_{N}}$, A is $\mathbf{Y}_{(n)}$, the mode- $n$ unfolding of the tensor $\mathcal{Y}$ obtained in (9). Consequently, $m=I_{n}, n=\prod_{k \neq n} R_{n}$, and the computational saving is huge when the tensor order $N$ or multilinear rank parameters $\left(R_{1}, R_{2}, \cdots, R_{N}\right)$ are large. In some particular cases, we may end up with a fat rectangular matrix, $\mathbf{Y}_{(n)}$ $(n>m)$. In this case, we can perform QRP on a square matrix, $\mathbf{Y}_{(n)} \mathbf{Y}_{(n)}^{T}$.

QRP Implementation. The QRP in our implementation is based on the Householder reflection. This method computes the orthogonal matrix $\mathbf{Q}$ as the product of multiple Householder reflection matrices:

$$
\mathrm{Q}=\mathrm{H}_{\mathbf{1}} \mathbf{H}_{2} \ldots \mathrm{H}_{\mathrm{m}-2} \mathrm{H}_{\mathrm{m}-\mathbf{1}} \text {. }
$$

The $j$-th reflection matrix, $\mathbf{H}_{j}$, is defined as:

$$
\mathbf{H}_{j}=\mathbf{I}-2 \mathbf{v}_{j} \mathbf{v}_{j}^{T}=\mathbf{I}-2 \frac{\mathbf{u}_{j} \mathbf{u}_{j}^{T}}{\mathbf{u}_{j}^{T} \mathbf{u}_{j}},
$$


TABLE III

PERFORMANCE COMPARISON OF FPGA AND CPU ON THE TTM TASK.

\begin{tabular}{|c|l|l|l|l|l|}
\hline \multirow{2}{*}{ Tensor Size } & \multirow{2}{*}{ Matrix Size } & \multicolumn{2}{|c|}{ CPU } & \multicolumn{2}{c|}{ FPGA } \\
\cline { 3 - 6 } & & Run-Time & Energy & Run-Time & Energy \\
\hline $32 \times 32 \times 32$ & $32 \times 32$ & $0.493 \mathrm{~ms}$ & $22.19 \mathrm{~mJ}$ & $0.148 \mathrm{~ms}$ & $0.4212 \mathrm{~mJ}$ \\
\hline $32 \times 32 \times 64$ & $32 \times 64$ & $0.596 \mathrm{~ms}$ & $26.82 \mathrm{~mJ}$ & $0.281 \mathrm{~ms}$ & $0.8000 \mathrm{~mJ}$ \\
\hline $32 \times 32 \times 128$ & $32 \times 128$ & $1.165 \mathrm{~ms}$ & $52.43 \mathrm{~mJ}$ & $0.546 \mathrm{~ms}$ & $1.556 \mathrm{~mJ}$ \\
\hline $32 \times 32 \times 256$ & $32 \times 256$ & $2.021 \mathrm{~ms}$ & $90.95 \mathrm{~mJ}$ & $1.077 \mathrm{~ms}$ & $3.067 \mathrm{~mJ}$ \\
\hline
\end{tabular}

TABLE IV

PERFORMANCE COMPARISON OF FPGA AND CPU ON THE KRONECKER PRODUCT TASK.

\begin{tabular}{|l|l|l|l|l|l|}
\hline \multirow{2}{*}{ Size of $\mathbf{x}_{j}$} & \multirow{2}{*}{ Size of $\mathbf{x}_{k}$} & \multicolumn{2}{|c|}{ CPU } & \multicolumn{2}{c|}{ FPGA } \\
\cline { 3 - 6 } & & Run-Time & Energy & Run-Time & Energy \\
\hline $1 \times 32$ & $1 \times 32$ & $9.655 \mu \mathrm{s}$ & $0.4345 \mathrm{~mJ}$ & $0.578 \mu \mathrm{s}$ & $2.111 \mu \mathrm{J}$ \\
\hline $1 \times 64$ & $1 \times 64$ & $14.72 \mu \mathrm{s}$ & $0.6624 \mathrm{~mJ}$ & $2.301 \mu \mathrm{s}$ & $8.403 \mu \mathrm{J}$ \\
\hline $1 \times 128$ & $1 \times 128$ & $24.87 \mu \mathrm{s}$ & $1.119 \mathrm{~mJ}$ & $9.195 \mu \mathrm{s}$ & $33.58 \mu \mathrm{J}$ \\
\hline $1 \times 256$ & $1 \times 256$ & $48.24 \mu \mathrm{s}$ & $2.171 \mathrm{~mJ}$ & $38.55 \mu \mathrm{s}$ & $140.7 \mu \mathrm{J}$ \\
\hline
\end{tabular}

where $\mathbf{u}_{j}$ is an unit vector and $\mathbf{u}_{j}=\frac{\mathbf{v}_{j}}{\left\|\mathbf{v}_{j}\right\|}$. Vector $\mathbf{v}_{j}$ can be chosen based the $j$ th column of $\mathbf{A}, \mathbf{a}_{j}$ :

$$
\mathbf{v}_{j}=\mathbf{a}_{j}+\operatorname{sign}\left(a_{j j}\right)\left\|\mathbf{a}_{n}\right\| \mathbf{e}_{1}
$$

During every iteration of QRP, we need to update $\mathbf{A}$ by multiplying it with the Householder matrix $\mathbf{H}$. In order to generate the permutation matrix, $\mathbf{P}$, we need to compare the norms of the columns of the updated matrix $\mathbf{A}$ at every iteration, arranging the columns so that the norms of the columns are in descending order. In this way, we can place the most weighted entries in the upper left corner of $\mathbf{Q}$, achieving the similar accuracy to SVD. Since we need to compare the norms of the columns at each iteration, the QRP operation is sequential. In other words, the comparison of the column norms made it very difficult to parallelize the algorithm on FPGA. Thus, we implement the Householder QR decomposition [30] with column pivoting on CPU.

\section{RESUlts}

This section shows the performance of our hybrid FPGACPU accelerator on both synthetic and real-world datasets. We first verify the performance of individual FPGA modules for the TTM and Kronecker product. Afterwards, we verify the performance of the whole FPGA-CPU sparse Tucker accelerator and compare it with CPU. We use the FPGA model XCVU9P-FLGA2577-3-e in our experiment. The maximum frequency of the FPGA implementation is $890 \mathrm{MHz}$. The CPU model used is Intel(R) Core(TM) i7-6820HK CPU @ $2.70 \mathrm{GHz}$. The size of the RAM is 16GB. The CPU has a maximum memory bandwith of $34.1 \mathrm{~GB} / \mathrm{s}$ and a thermal design power (TDP) of $45 \mathrm{~W}$. In the experiments, we prioritize the computations on CPU to achieve the maximum performance, therefore, the energy consumption on CPU can be estimated as the product of runtime and TDP. We estimate the energy cost of sparse Tucker decomposition on FPGA on Xilinx Vivado via Amazon Web Service. The communication protocol between FPGA and CPU is PCI express, which has a maximum bandwidth of $10 \mathrm{~GB} / \mathrm{s}$. Our design can also be implemented on a low-end FPGA such as Zynq-7100 as well. On a low-end FPGA, We may decrease the LUT utilization by adjusting the unroll factor in our TTM module implementation.

\section{A. Performance of Individual FPGA Modules}

Firstly we verify the performance of the TTM and Kronecker-Product modules on some synthetic tensor data, and summarize their performance below:
- TTM Module: We verify the performance by considering a set of 3-way tensors $\mathcal{Y} \in \mathbb{R}^{R_{1} \times R_{2} \times I_{3}}$ and factor matrices $\mathbf{U} \in \mathbb{R}^{R_{3} \times I_{3}}$. The rank of approximate, $R_{1}$, $R_{2}$ and $R_{3}$, are always very small compared with the original tensor size for data compression. Thus, we set $R_{1}=R_{2}=R_{3}=32$. The original tensor size, $I_{3}$ is set to increase from 32 to 256 as shown in Table IIII In the reallife examples, the original tensor size $I_{3}$ can definitely be larger than 256. And the performance of the tensortimes-matrix (TTM) module won't perform significantly worse when the original tensor size becomes extremely large. Here, we set the maximum of our tensor size to be 256 for experimental purpose only. The FPGA achieves $1.560 \times$ to $3.331 \times$ speedup than CPU on these tensormatrix products. We also compare the energy consumption between FPGA and CPU on the tensor-times-matrix task. As shown in Table III] the FPGA saves $95.6 \%$ to $98.1 \%$ of energy compared with CPU.

- Kronecker-Product Module: As shown in Section 4.3, the Kronecker product used in the sparse Tucker decomposition deals with two row vectors, $\mathbf{x}_{j} \in \mathbb{R}^{1 \times R_{j}}$ and $\mathbf{x}_{k} \in \mathbb{R}^{1 \times R_{k}}$. Therefore, we compare the performance of Kronecker products on FPGA and CPU by changing the rank parameters $R_{1}$ and $R_{2}$ from 32 to 256 . The rank of approximation $R_{1}$ and $R_{2}$ does not necessarily need to be equal to each other. We set $R_{1}$ and $R_{2}$ to be equal for experimental purpose only. In addition, the rank of approximation $R_{1}, R_{2}$ and $R_{3}$ are usually very small compared with the original tensor size for data compression. We increase the rank from 32 to 256 to demonstrate the performance of Kronecker product module. We estimated the power of the CPU to be $45 \mathrm{~W}$. The energy consumption of CPU is estimated by multiplying the power with the CPU time. The results are shown in Table IV] The speedup of FPGA over CPU ranges from $1.251 \times$ to $16.704 \times$. As shown in Table IV] FPGA consumes $93.519 \%$ to $99.514 \%$ less energy than CPU on the Kronecker-product tasks.

\section{B. Accelerator's Performance: Synthetic Datasets}

Now we evaluate the whole hybrid FPGA-CPU accelerator on some randomly generated synthetic sparse tensor data sets. Specifically, we consider a set of 3-way tensors $\mathcal{X} \in \mathbb{R}^{200 \times 200 \times 200}$ with different sparsity. We fix the rank parameters $R_{1}=R_{2}=R_{3}=16$.

Fig. 6 compares the run-time of our hybrid FPGA-CPU platform with CPU and densor FPGA accelerator [25]. The 


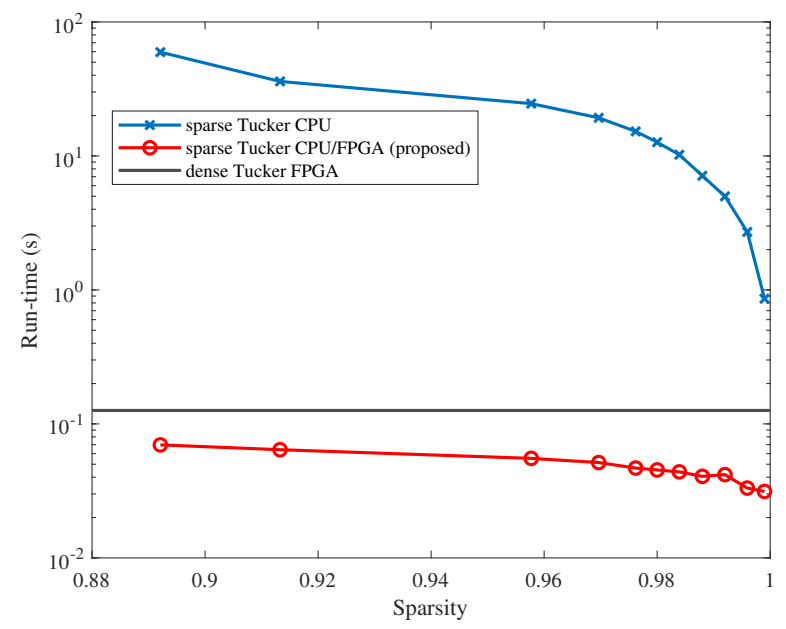

Fig. 6. Run-time comparison between the proposed hybrid platform, dense FPGA accelerator and CPU on a set of $200 \times 200 \times 200$ synthetic random tensors with different sparsity.

speedup of the hybrid FPGA-CPU accelerator is $27 \times \sim 853 \times$ compared with CPU. The speedup of our sparse Tucker accelerator is $1.167 \times \sim 126 \times$ faster than the FPGA accelerator designed for dense Tucker decompsition [25]. In the whole sparse Tucker decomposition algorithm, the Kronecker product module takes the most amount of time. However, this module is parallelized in our design, and it is significantly sped up on FPGA as shown in Section IV-A. When the tensor has more non-zero elements, more Kronecker-product operations are required, leading to a more significant speedup on FPGA.

\section{Real-World Datasets}

Finally, we verify our accelerator on four real-world sparse tensor data sets [34]-[37]. In addition, we compare the performance of our accelerator with sparse Tucker decomposition on CPU and with the dense FPGA accelerator in [25]. Table V shows the detailed run-time and energy consumption of different methods on these datasets. TableVI further shows the overall hardware resource utilization of our method on FPGA. The FPGA design is compiled for each data set in order to achieve the maximum efficiency. We use BRAM_18K, BDSP48E, FF and LUT to denote block random access memory, digital signal processing elements, flip flops and lookup tables, respectively.

The detailed experiments and results are summarized below:

- Amazon Reviews Datasets [34]. The modes of this threeway tensor represent users, products, and words, respectively. Each non-zero element in this tensor is the number of times a word appears in a given review. Additionally, we extract one portion of the Amazon reviews tensor of size $20000 \times 20000 \times 20000$ and choose the rank of approximation as $R_{1}=R_{2}=R_{3}=32$. We perform 2 power iterations on all modes. The sizes of the tensors and matrices in TTM (12) are $32 \times 32 \times 20000$ and $32 \times 20000$, respectively. This sparse Tucker factorization involves 9 calls of QR decomposition on a set of $20000 \times 32$ matrices in total to compute the orthogonal factor matrices. Finally, there are totally 8,820 calls of Kronecker products, which depends on the number of non-zero tensor entries. On this dataset, our hybrid FPGA/CPU platform achieves $1.15 \times$ speedup than CPU with only $13.5 \%$ energy consumption. Our method also achieves $1091 \times$ speedup than the dense Tucker FPGA accelerator [25].
- NELL-2 Datasets [37]. This data set is extracted from the Never Ending Language Learner knowledge base. The non-zero entries represent some entity-relation-entity tuples. We extract one portion of the NELL-2 data set and obtain a sparse tensor of size $1000 \times 1000 \times 1000$. In addition, we choose our rank of approximation as $R_{1}=R_{2}=R_{3}=16$. We perform 5 power iterations on all modes. The sizes of the tensors and matrices in TTM (12) are $16 \times 16 \times 1000$ and $16 \times 1000$, respectively. This sparse Tucker factorization involves 15 calls of QR decomposition on a set of $1000 \times 256$ matrices in total to compute the orthogonal factor matrices. Finally, there are totally 432,555 calls of Kronecker products, which depends on the number of non-zero tensor entries. Our hybrid FPGA/CPU platform achieves $18 \times$ speedup and $94.8 \%$ energy saving compared with CPU. Our method is also $23.6 \times$ faster than the dense FPGA accelerator [25].

- Binary 3-Way Tensor for Parallel Matrix Multiplication [35], [36]. This binary tensor describes the parallel computation process of matrix multiplications. Given two matrices $\mathbf{A} \in \mathbb{R}^{M \times K}$ and $\mathbf{B} \in \mathbb{R}^{K \times N}$, their product results in a matrix $\mathbf{C} \in \mathbb{R}^{M \times N}$. Let $I_{1}=M K$, $I_{2}=K N$ and $I_{3}=M N$, then a binary 3-way tensor $\mathcal{X}$ can represent the parallel matrix multiplication. The first mode corresponds to the first input matrix $\mathbf{A}$ with entries in row-major order; the second mode corresponds to the input matrix $\mathbf{B}$ with entries in row-major order; the third mode corresponds to the output matrix $\mathbf{C}$ with entries in column-major order. A nonzero entry $x_{i_{1} i_{2} i_{3}}=1$ corresponds to a scalar multiplication within the classical matrix multiplication algorithm: the $i_{1}$-th entry of $\mathbf{A}$ is multiplied with the $i_{2}$-th entry of $\mathbf{B}$, and the result is accumulated into the $i_{3}$-th entry of $\mathbf{C}$. The number of nonzero elements in $\mathcal{X}$ is $n n z=M K N$. We consider the case $M=N=K=5$, which results in a binary tensor $\mathcal{X}$ with size $25 \times 25 \times 25$ and a sparsity of $8 \times 10^{-3}$. To perform sparse Tucker decomposition on this 3-way binary tensor, we choose an approximation rank of $R_{1}=R_{2}=R_{3}=5$. We perform three steps of high-order power iterations on all modes, leading to 3 TTM in (12) and totally 6 calls for QR decomposition with column pivoting. Finally, the number of Kronecker products used in this data set is 1,125 . Our meethod achieves $37 \times$ and $1.52 \times$ speedup than CPU and than the dense FPGA accelerator [25], respectively. Compared with the sparse Tucker decomposition on CPU, our accelerator saves $97.1 \%$ energy.

- Retinal Angiogram. Angiogramy is a medical diagnoictic test that uses X-ray to take picture of the blood vessels. The images, angiogram, are always very sparse. Fig. 6 shows the retinal angiogram of a patient on the left. The size of the original retinal angiogram is $130 \times 150$ [38]. Tucker factorization can also be employed to compress 2-D data, because a matrix is the special case of a tensor. Different from SVD compression of a matrix where the rank is a scalar, a Tucker decomposition allows one to set two rank parameters. We perform a sparse Tucker decomposition with rank $\mathrm{R}=[30,35]$ on this image. We performed 12 steps of high-order power iterations on all modes, leading to 12 TTM in (12) and totally 24 calls for QR decomposition with column pivoting. We do not need any Kronecker products since the order of the tensor is 2 . Our proposed method achieves $19 \times$ speedup than CPU and $1.91 \times$ speedup than dense FPGA accelerator [25], and it saves $94.4 \%$ energy compared 
TABLE V

PERFORMANCE OF SPARSE TUCKER DECOMPOSITION ON REAL-WORLD BENCHMARKS.

\begin{tabular}{|c|c|c|c|c|c|}
\hline \multicolumn{2}{|l|}{ Benchmarks } & Amazon & Nell-2 & Parallel Matrix Multiplication & Retinal Angiogram \\
\hline \multicolumn{2}{|l|}{ Tensor Size } & $20 K \times 20 K \times 20 K$ & $1 K \times 1 K \times 1 K$ & $25 \times 25 \times 25$ & $130 \times 150$ \\
\hline \multicolumn{2}{|l|}{ Sparsity } & $1.128 \times 10^{-10}$ & $2.40 \times 10^{-5}$ & $8 \times 10^{-3}$ & 0.18 \\
\hline \multirow{2}{*}{$\mathrm{CPU}$} & Run-Time & $100.045 \mathrm{~s}$ & $7.355 \mathrm{~s}$ & $8.175 \times 10^{-2} \mathrm{~s}$ & $0.1838 \mathrm{~s}$ \\
\hline & Energy & $4502.03 \mathrm{~J}$ & $330.98 \mathrm{~J}$ & $3.68 \mathrm{~J}$ & $8.27 \mathrm{~J}$ \\
\hline \multirow{2}{*}{$\begin{array}{l}\text { Hybrid FPGA/CPU } \\
\text { (proposed) }\end{array}$} & Run-Time & $86.785 \mathrm{~s}$ & $0.403 \mathrm{~s}$ & $2.179 \times 10^{-3} \mathrm{~s}$ & $9.898 \times 10^{-3} \mathrm{~s}$ \\
\hline & Energy & $3896.08 \mathrm{~J}$ & $17.10 \mathrm{~J}$ & $0.1057 \mathrm{~J}$ & $0.4667 \mathrm{~J}$ \\
\hline Dense FPGA Tucker $[25]$ & Run-Time & $9.47 \times 10^{4} \mathrm{~s}$ & $9.5 \mathrm{~s}$ & $9.9 \times 10^{-3} \mathrm{~s}$ & $1.18 \times 10^{-2} \mathrm{~s}$ \\
\hline
\end{tabular}

TABLE VI

UTILIZATION OF FPGA ON REAL-WORLD BENCHMARKS. IN THE COLUMN OF "MEMORY" WE LIST THE NUMBER OF BRAM, WHERE EACH BRAM HAS $18 \times 10^{3}$ BITS

\begin{tabular}{|c|c|c|c|c|c|c|c|c|c|}
\hline \multicolumn{2}{|c|}{ Name } & Expression & Instance & Memory & Multiplexer & Register & Total & Available & Utilization (\%) \\
\hline \multirow{4}{*}{ Amazon } & BRAM_18K & - & - & 542 & - & - & 542 & 4320 & 13 \\
\hline & DSP4 $\overline{48 E}$ & - & 282 & - & - & - & 282 & 6840 & 4 \\
\hline & $\mathrm{FF}$ & 0 & 17257 & - & - & 107670 & 124927 & 2364480 & 5 \\
\hline & LUT & 406251 & 17649 & - & 20587 & - & 443268 & 1182240 & 37 \\
\hline \multirow{4}{*}{ Nell-2 } & BRAM_18K & - & - & 63 & - & - & 63 & 4320 & 1 \\
\hline & DSP48E & - & 470 & - & - & - & 470 & 6840 & 7 \\
\hline & $\mathrm{FF}$ & 0 & 29495 & - & - & 54691 & 84186 & 2364480 & 4 \\
\hline & LUT & 405656 & 30863 & - & 13972 & - & 450491 & 1182240 & 38 \\
\hline \multirow{4}{*}{$\begin{array}{c}\text { Parallel } \\
\text { Matrix } \\
\text { Multiplication }\end{array}$} & BRAM_18K & - & - & 2 & - & - & 2 & 4320 & $\sim 0$ \\
\hline & DSP48E & - & 16 & - & - & - & 16 & 6840 & $\sim 0$ \\
\hline & $\mathrm{FF}$ & 0 & 759 & - & - & 107 & 866 & 2364480 & $\sim 0$ \\
\hline & LUT & 49799 & 778 & - & 707 & - & 51284 & 1182240 & 4 \\
\hline \multirow{4}{*}{$\begin{array}{c}\text { Retinal } \\
\text { Angiogram }\end{array}$} & BRAM_18K & - & - & 5 & - & - & 5 & 4320 & $\sim 0$ \\
\hline & DSP48E & - & 21 & - & - & - & 21 & 6840 & $\sim 0$ \\
\hline & $\mathrm{FF}$ & 0 & 1171 & - & - & 9438 & 10609 & 2364480 & $\sim 0$ \\
\hline & LUT & 121303 & 1089 & - & 2256 & - & 124648 & 1182240 & 11 \\
\hline
\end{tabular}
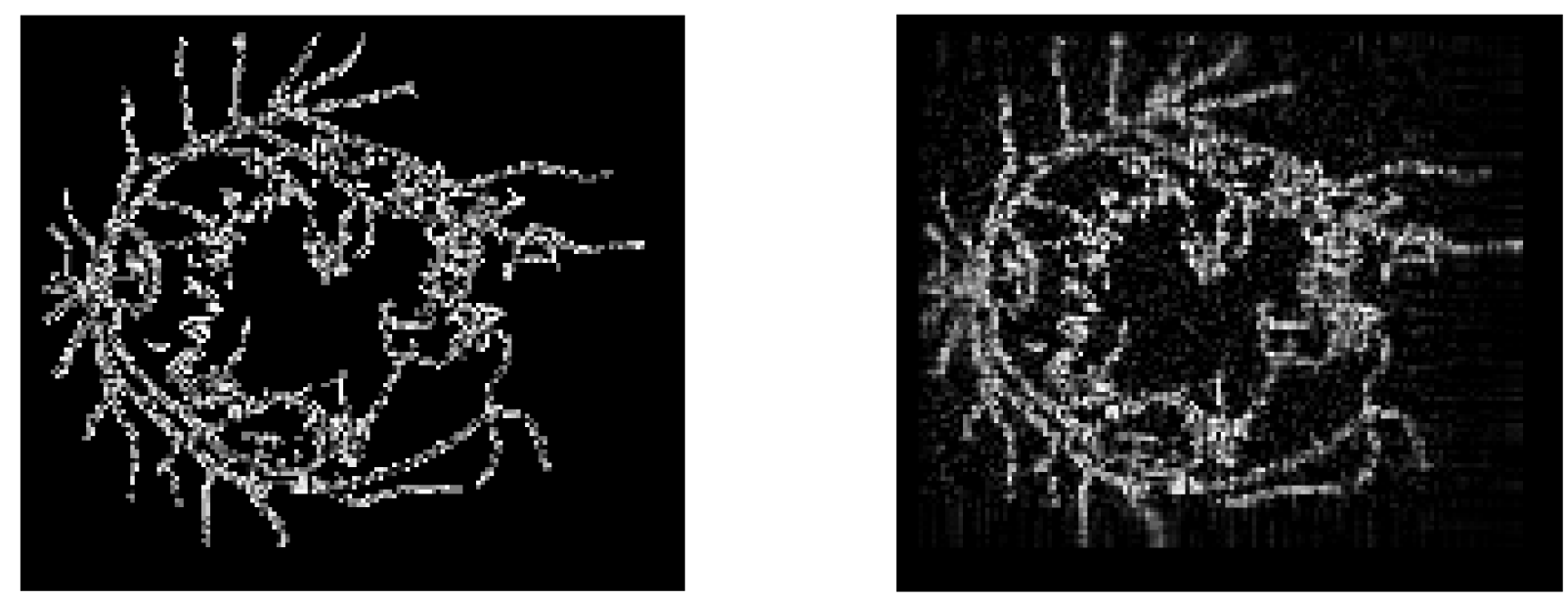

Fig. 7. Left: the original retinal angiogram. Right: the approximated image by our sparse Tucker decomposition on the FPGA/CPU hybrid platform.

with the sparse Tucker factorization on CPU. Fig. 7 compares the original retinal angiogram and the resulting compressed image from our FPGA/CPU hybrid accelerator. The compression ratio is $18.57 \times$. While the image is highly compressed, the essential features, such as blood vessels, are still clearly preserved.

\section{CONCLUSION}

This paper has proposed a hybrid FPGA-CPU accelerator for sparse Tucker decomposition. On the algorithm level, the Kronecker products have exploited the data sparsity and has significantly reduced the computational complexity. The QR with pivoting method have dramatically reduced the complexity of obtaining the orthogonal mode-n matrix factors. The FPGA modules for the tensor-times-matrix and for the Kronecker products have achieved $93.519 \%$ to $99.514 \%$ energy saving compared with CPU on synthetic benchmarks. The proposed hybrid FPGA-CPU accelerator has been evaluated with both synthetic and realistic sparse tensor data sets. It has achieved $27 \times \sim 853 \times$ speedup over CPU and $1.167 \times \sim 126 \times$ speedup over the recently developed dense Tucker FPGA accelerator [25] on the synthetic datasets. Our proposed methods have also achieved $1.15 \times \sim 1091 \times$ speedup and over $95 \%$ energy savings on the tested real-world tensor datasets. Our proposed accelerator have significantly outperformed CPU and dense Tucker FPGA accelerator [25] when the tensor is very large and sparse.

\section{REFERENCES}

[1] T. G. Kolda and B. W. Bader, "Tensor decompositions and applications," SIAM review, vol. 51, no. 3, pp. 455-500, 2009. 
[2] I. Oseledets and E. Tyrtyshnikov, "Tt-cross approximation for multidimensional arrays," Linear Algebra and its Applications, vol. 432, no. 1, pp. $70-88,2010$.

[3] L. De Lathauwer, B. De Moor, and J. Vandewalle, "A multilinear singular value decomposition," SIAM journal on Matrix Analysis and Applications, vol. 21 , no. 4, pp. 1253-1278, 2000

[4] - "On the best rank-1 and rank- $\left(r_{1}, r_{2}, \ldots, r_{n}\right)$ approximation of higher-order tensors," SIAM journal on Matrix Analysis and Applications, vol. 21 , no. 4, pp. 1324-1342, 2000

[5] J. D. Carroll and J. J. Chang, "Analysis of individual differences in multidimensional scaling via an n-way generalization of "Eckart-Young" decomposition," Psychometrika, vol. 35, no. 3, pp. 283-319, 1970.

[6] R. A. Harshman, "Foundations of the PARAFAC procedure: Models and conditions for an "explanatory" multi-modal factor analysis," UCLA Working Papers in Phonetics, vol. 16, no. 1, p. 84, 1970.

[7] M. A. O. Vasilescu and D. Terzopoulos, "Multilinear analysis of image ensembles: Tensorfaces," in European Conference on Computer Vision. Springer, 2002, pp. 447-460.

[8] 1 , "Multilinear subspace analysis of image ensembles," in IEEE Prof. Computer Vision and Pattern Recognition, vol. 2, 2003, pp. II-93.

[9] - "Multilinear independent components analysis," in IEEE Conf. Computer Vision and Pattern Recognition, vol. 1, 2005, pp. 547-553.

[10] T. G. Kolda and J. Sun, "Scalable tensor decompositions for multi-aspect data mining," in Prof. IEEE Int. Conf. Data Mining, 2008, pp. 363-372.

[11] N. Batmanghelich, A. Dong, B. Taskar, and C. Davatzikos, "Regularized tensor factorization for multi-modality medical image classification," in International Conference on Medical Image Computing and ComputerAssisted Intervention. Springer, 2011, pp. 17-24.

[12] Z. Zhang, T.-W. Weng, and L. Daniel, "Big-data tensor recovery for high-dimensional uncertainty quantification of process variations," IEEE Transactions on Components, Packaging and Manufacturing Technology, vol. 7, no. 5, pp. 687-697, 2016.

[13] Z. Zhang, X. Yang, I. V. Oseledets, G. E. Karniadakis, and L. Daniel, "Enabling high-dimensional hierarchical uncertainty quantification by anova and tensor-train decomposition," IEEE Trans. CAD of Integrated Circuits and Systems, vol. 34, no. 1, pp. 63-76, 2014.

[14] Z. Zhang, K. Batselier, H. Liu, L. Daniel, and N. Wong, "Tensor computation: A new framework for high-dimensional problems in eda," IEEE Transactions on Computer-Aided Design of Integrated Circuits and Systems, vol. 36, no. 4, pp. 521-536, 2016.

[15] J. Luan and Z Zhang, "Prediction of multi-dimensional spatial variation data via bayesian tensor completion," IEEE Transactions on ComputerAided Design of Integrated Circuits and Systems, 2019.

[16] A. Novikov, D. Podoprikhin, A. Osokin, and D. P. Vetrov, "Tensorizing neural networks," in Advances in Neural Information Processing Systems, 2015, pp. 442-450.

[17] Y. Yang, D. Krompass, and V. Tresp, "Tensor-train recurrent neural networks for video classification," in Proc. Int. Conf. Machine Learning, vol. 70, 06-11 Aug 2017, pp. 3891-3900.

[18] C. Hawkins and Z. Zhang, "Bayesian tensorized neural networks with automatic rank selection," arXiv preprint arXiv:1905.10478, 2019.

[19] O. Kaya and B. Uçar, "High performance parallel algorithms for the tucker decomposition of sparse tensors," in 2016 45th International Conference on Parallel Processing (ICPP). IEEE, 2016, pp. 103-112.

[20] S. Smith, J. Park, and G. Karypis, "Sparse tensor factorization on many-core processors with high-bandwidth memory," in Proc. IEEE Int. Parallel and Distributed Processing Symp, 2017, pp. 1058-1067.

[21] J. Li, C. Battaglino, I. Perros, J. Sun, and R. Vuduc, "An inputadaptive and in-place approach to dense tensor-times-matrix multiply," in Proc. Int. Conf. High Performance Computing, Networking, Storage and Analysis, 2015, pp. 1-12

[22] Y.-D. Kim, E. Park, S. Yoo, T. Choi, L. Yang, and D. Shin, "Compression of deep convolutional neural networks for fast and low power mobile applications," arXiv preprint arXiv:1511.06530, 2015.

[23] N. Srivastava, H. Rong, P. Barua, G. Feng, H. Cao, Z. Zhang, D. Albonesi, V. Sarkar, W. Chen, P. Petersen et al., "T2s-tensor: Productively generating high-performance spatial hardware for dense tensor computations," in International Symp. Field-Programmable Custom Computing Machines (FCCM), 2019, pp. 181-189.

[24] W.-P. Huang, B. P. Kwan, W. Ding, B. Min, R. C. Cheung, L. Qi, and H. Yan, "High performance hardware architecture for singular spectrum analysis of hankel tensors," Microprocessors and Microsystems, vol. 64 pp. 120-127, 2019.

[25] K. Zhang, X. Zhang, and Z. Zhang, "Tucker tensor decomposition on fpga," in Intl. Conf. Computer Aided Design, no. 6A.1, 2019.

[26] N. Srivastava, H. Jin, S. Smith, H. Rong, D. Albonesi, and Z. Zhang, "Tensaurus: A versatile accelerator for mixed sparse-dense tensor computations," in 2020 IEEE International Symposium on High Performance Computer Architecture (HPCA). IEEE, 2020, pp. 689-702.

[27] S. F. Roohi, D. Zonoobi, A. A. Kassim, and J. L. Jaremko, "Dynamic mri reconstruction using low rank plus sparse tensor decomposition," in IEEE Int. Conf. Image Processing, 2016, pp. 1769-1773.

[28] P. Fillard, V. Arsigny, X. Pennec, P. M. Thompson, and N. Ayache, "Extrapolation of sparse tensor fields: Application to the modeling of brain variability," in Biennial International Conference on Information Processing in Medical Imaging. Springer, 2005, pp. 27-38.
[29] C. F. Van Loan, "The ubiquitous kronecker product," Journal of computational and applied mathematics, vol. 123, no. 1-2, pp. 85-100, 2000.

[30] G. H. Golub and C. F. V. V. Loan, Matrix Computations, 3rd ed. Baltimore, MD: Johns Hopkins University Press, 1996.

[31] G. H. Golub and C. Reinsch, "Singular value decomposition and leas squares solutions," in Linear Algebra. Springer, 1971, pp. 134-151.

[32] F. G. Gustavson, "Some basic techniques for solving sparse systems of linear equations," in Sparse matrices and their applications. Springer, 1972, pp. 41-52.

[33] P. A. Tew, "An investigation of sparse tensor formats for tensor libraries," Ph.D. dissertation, Massachusetts Institute of Technology, 2016.

[34] J. McAuley and J. Leskovec, "Hidden factors and hidden topics: understanding rating dimensions with review text," in Proceedings of the 7th ACM conference on Recommender systems. ACM, 2013, pp. $165-172$.

[35] A. R. Benson and G. Ballard, "A framework for practical parallel fast matrix multiplication," in Proc. ACM SIGPLAN Symposium on Principles and Practice of Parallel Programming, 2015, pp. 42-53.

[36] R. P. Brent, "Algorithms for matrix multiplication," Stanford University, Tech. Rep., 1970.

[37] A. Carlson, J. Betteridge, B. Kisiel, B. Settles, E. R. Hruschka Jr., and T. M. Mitchell, "Toward an architecture for never-ending language learning." in AAAI, vol. 5, 2010, p. 3.

[38] A. Hoover, V. Kouznetsova, and M. Goldbaum, "Locating blood vessels in retinal images by piecewise threshold probing of a matched filter response," IEEE Transactions on Medical imaging, vol. 19, no. 3, pp. 203-210, 2000

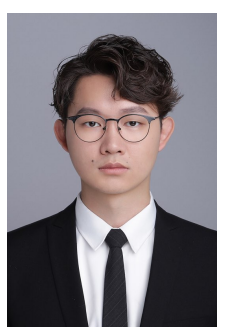

Weiyun Jiang received his B.Sc. degree in Electrical Engineering from University of California at Santa Barbara, Santa Barbara, CA, in 2020. Currently he is a graduate student of Electrical Engineering at Stanford University, Stanford, CA. His research interests include algorithm/hardware co-design for tensor data analysis and machine learning.

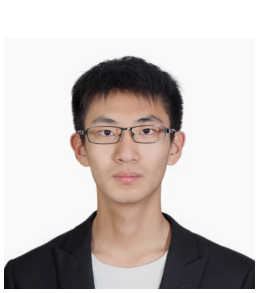

Kaiqi Zhang received the B.Sc. degree in electronic engineering from Tsinghua University, Beijing, China, in 2016, the M.S. degree in electrical and computer engineering from the University of California at Davis, Davis, CA, in 2018, and he is now pursuing his Ph.D. degree in electrical and computer engineering from the University of California at Santa Barbara, Santa Barbara, CA.

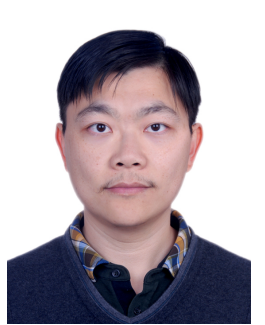

Colin Yu Lin received his B.Sc. degree in electronic engineering from Sun Yat-Sen University, Guangzhou, China, in 2005, the M.E. degree in computer engineering from the University of Chinese Academy of Sciences, Beijing, China, in 2008, and the $\mathrm{Ph} . \mathrm{D}$. degree in electrical and electronic engineering from the University of Hong Kong, Hong Kong, in 2012. From 2011 to 2012, he was a Visiting Student Researcher with the Department of Electrical Engineering and Computer Sciences and the Berkeley Wireless Research Center, University of California at Berkeley, Berkeley, CA, USA. He was an Assistant Professor with the System on Programmable Chip Research Department, Institute of Electronics, Chinese Academy of Sciences, Beijing, China, from 2012 to 2016. He is currently a Software Development Senior Manager with Data Center Group, Xilinx, Inc. His current research interests include FPGA architecture, CAD for FPGAs, high level synthesis, and FPGA for high performance computing. 


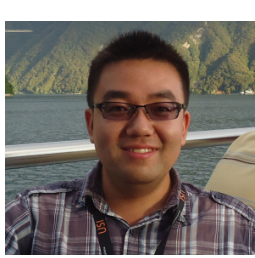

Feng Xing received his B.Sc degree from Wuhan University, China and M.Sc from University of Lille,

French on pure mathematics and applied mathematics. He got his Ph.D. from "Maison de la Simulation", CEA Saclay on high performance computing in 2014. Then he worked as postdoc researcher at INRIA French and BRGM French for two years on high performance geothermal simulation. He is currently a software development manager at Xilinx, Inc.

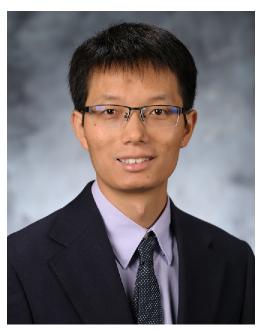

Zheng Zhang (M'15) has been an Assistant Professor of Electrical and Computer Engineering with the University of California at Santa Barbara (UCSB), since July 2017. He received his Ph.D in Electrical Engineering and Computer Science from Massachusetts Institute of Technology (MIT), Cambridge, MA, in 2015. His research interests include uncertainty quantification and tensor computation. The applications of his research include design automation of nano-scale electronics and photonics, algorithm/hardware co-design of high-dimensional, robust and safe machine learning systems.

Dr. Zhang received three best paper awards from IEEE Transactions: the Best Paper Award of IEEE Transactions on Computer-Aided Design of Integrated Circuits and Systems in 2014, two Best Paper Awards of IEEE Transactions on Components, Packaging and Manufacturing Technology in 2018 and 2020, respectively. He also received three Best Paper Awards at international conferences. His PhD dissertation won the ACM SIGDA Outstanding Ph.D Dissertation Award in Electronic Design Automation in 2016, and the Best Thesis Award from the Microsystems Technology Laboratory of MIT in 2015. He received a NSF CAREER Award in 2019 and a Facebook Research Award in 2020 